\title{
SCATTERED LIGHT IN SPECTROPHOTOMETRY AND A NEW FORM OF SPECTROPHOTOMETER.
}

By Herbert E. Ives.

RECENTLY, in making some spectrophotometric measurements of artificial light sources in terms of daylight, the writer's attention was called to the importance of scattered light as a source of error in spectrophotometry. Scattered light is practically impossible to avoid in optical instruments. For this reason it is surprising that it receives next to no attention in the design or the published descriptions of the manipulation of spectrophotometers. In such instruments as the Brace and Lummer-Brodhun diaphragms in the collimator and telescope tubes constitute the sum of the precautions taken to avoid stray light. Yet under certain circumstances, to be outlined below, such precautions are insufficient; in fact, only by the use of other forms of instruments can the stray light error be entirely eliminated.

The first essential of a spectrophotometer is complete symmetry; the light from the two sources must be subject to identical treatment in its passage through the instrument to the eye. This symmetry must hold not only when the two sources are identical, but when they are different in special character. It is in this latter requirement that some of the present forms are deficient. Scattered light, when present, is present usually in different quantity and quality in the fields due to the two sources. This deficiency becomes apparent usually only on measuring light sources as different in character as daylight and the yellow artificial illuminants, in which case considerable errors are possible.

The source of this scattered light, its amount in different types of spectrophotometers, and the means to obviate it, form the subject of the present note.

In a spectrophotometer with a perfect system of diaphragms the remaining scattered light - visible as an illumination of the in- 
strument field if monochromatic light is used and the eye is observing at another wave-length - is due to light from the lens and prism surfaces, either from dust and scratches or from "ghosts." The peculiarity of this light (and also scattered light due to imperfect diaphragms) is that it is proportional not to the intensity of the wave-length under observation but to the total amount of light of all colors striking the scattering surface. When making observations at the ends of the spectrum, for instance, what one observes, even in the newest and best instruments, will be the deep red or blue superposed on a background of gray due to scattering. The older the instrument, the more dust or film on lens surfaces, or the more numerous the lens ghosts, the larger the proportion of the total light is this scattered background. This is especially noticeable in a spectrophotometer if a large quantity of light is used in an attempt to secure measurements in the fainter portions of the spectrum.

Now this scattered light can or cannot cause errors, depending on the following principle: If the scattered light is spread equally over the two photometric fields to be compared, it is harmless; if it is present on one side alone it results in error. In the former case a loss of photometric sensibility results which is not desirable, but is far less undesirable than the error which arises in the latter case from comparing the colored light of one side with the colored light plus scattered light of the other.

In undertaking the measurements above referred to the writer had available a Lummer-Brodhun spectrophotometer and a spectrometer converted into a spectrophotometer by the use of a right angle prism device at the slit. The chief difference in the instruments - which are representatives of two usual types of spectrophotometers - is that in the Lummer-Brodhun the spectra are focused on the eye; hence the prism face is seen of one color. In the other instrument two adjacent spectra are viewed by an eyepiece. In the Lummer-Brodhun instrument the division of the beams of light from the two sources is effected by the use of two collimators, and the photometric field is a Lummer-Brodhun prism between the collimator and the dispersing prism. In the other instrument the two beams of light are divided at the slit, but pass over identical 
[Vol. XXX.

paths through one collimator to the image plane of the telescope.

Were there no other factors to be considered than ease of observation the Lummer-Brodhun instrument would have been chosen, because the large monochromatic surface and extended dividing lines which are observed in that type permit of more accurate settings than does the narrow strip from the adjacent spectra on which comparisons are made in the adapted spectrometer. When, however, observations were made on two sources with a steep spectral gradient with respect to each other, for instance a light weak in red and strong in blue, against one strong in red and weak in blue, the following condition was found: At the red end of the spectrum it was necessary to admit so much of the light of the red deficient source that a mist of gray light was caused on the corresponding field by the large amount of the green and blue admitted. At the blue end the scattered light was in the field corresponding to the other source. At each end of the spectrum, therefore, one of the fields was grayer than the other and an intensity match would not be a true one. This grayness, it should be noted, may easily be confused with the lack of color match caused by very wide slits, with sources of steep spectral gradient. The effect of scattered light of this kind, present on one side of the instrument and not on the other, is in general to make the two sources measured appear nearer alike in character. Anomalous results at the ends of the spectrum may also be expected.

Attempts to eliminate the unsymmetrical illumination on the two sides met with partial success. By having the collimator lenses and Lummer-Brodhun prism scrupulously clean and by providing additional diaphragms outside the slits, large enough to place all the interior diaphragms in shadow, considerable improvement was produced. By using colored glasses of tints to absorb the portion of the spectrum not being observed (this, of course, changed the values of the readings) the improvement was still greater, and for most purposes this method would probably be perfectly satisfactory. It was, however, decided to use the instrument giving two juxtaposed spectra because the scattered light trouble appeared to be entirely absent. Scattered light was present; but with this important distinction, that the light forming the two spectra, in its 
passage to the image had filled the whole surfaces of all the lenses and prisms, hence the scattered light was spread equally over both images and the dividing line, thereby merely resulting in a loss of sensibility. Interposition of a colored glass at the ends of the spectrum made no difference in the values of the readings. There was, therefore, no uncertainty as to whether the disturbing factor had been entirely eliminated or in the periods between observations had changed in amount.

As mentioned above, the spectrophotometer in which two adjacent spectra are viewed is not sensitive because the dividing edge for any strip of the spectra such as could be compared, is extremely narrow. On the other hand, in existing designs of spectrophotometers in which the prism face is observed by monochromatic light the two beams of light usually pass through two separate collimators. In all, the prism, biprism or other device forming the photometric field, is a material object. It therefore separates not only the direct but the scattered light of each half of the field from the other half. In this class belong the Lummer-Brodhun and the Brace instruments. The Koenig spectrophotometer which separates the light partly at the slit and partly by a biprism, after passage through the single collimator, lies between the two classes. ${ }^{1}$

In an attempt to secure the advantage of both styles of spectrophotometer a new design has recently been constructed. The principles of this are: (1) That the light from both sources should go through one collimator and dispersive system in such manner that all surfaces which could cause scattering would receive light from both sources equally. (2) That the photometric plane should be a virtual one in space, through which the background of scattering could be seen behind each field equally, no matter from which side the scattered light originated.

$A$, Fig. 1 , is a Hilger constant deviation wave-length spectrometer. At 1 is placed any convenient device for forming a divided photometric field, such as a plaster-of-paris wedge, or a LummerBrodhun contrast prism. As set up a large and small right angle prism were cemented together, the face $b$ being entirely silvered,

\footnotetext{
${ }^{1}$ The writer has had no opportunity to work with a Koenig spectrophotometer, but study of its construction would indicate it to be practically free from the possibility of scattered light asymmetry.
} 
[VOL. XXX.

the face $a$ silvered half way up. 6 and 7 are the two sources to be compared, which must be extended, preferably white surfaces illuminated by the lights compared. At 2 (the slit) is a convex lens,

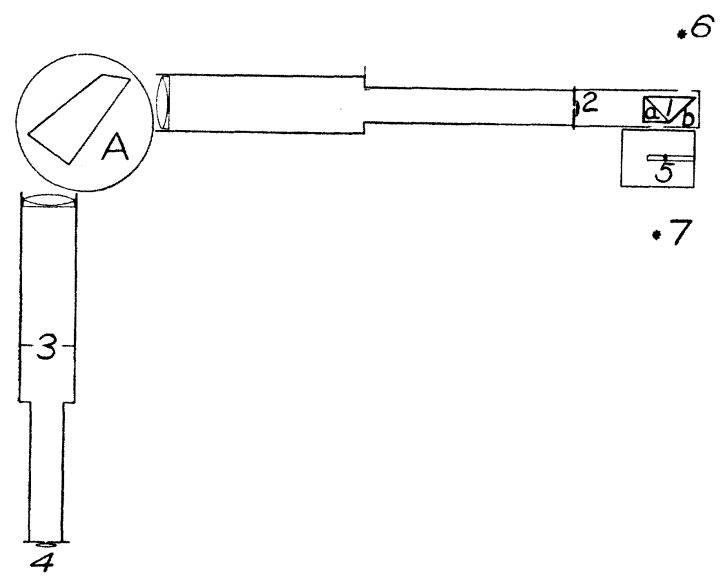

Fig. 1.

where distance and focal length are such that it forms, with the collimator and telescope lenses, an image of the prism face $a$ at some place in the telescope tube 3 , as distant as possible from the lenses and prism of the instrument. 5 is a Brodhun sector disc for reducing the intensity of one source by a measurable amount. At 4 is a spectacle lens to focus the image at 3 .

Now, if the dividing line at $a$ is perpendicular to the length of the slit at 2 a sharp image of it will be formed at 3 with any slit width practicable to work with. (Because of diffraction, lines parallel to the slit lose their sharpness with a very narrow slit.) Further, if the slit of the spectrophotometer be made long (as long as the diameter of the collimator lens for the best effect) the beam of light coming from any part of $a$ is spread over a large area of lenses and prisms, and if dust or scratches be present to cause scattering, the scattered light is seen as a background to the image at 3 . By properly choosing the length of slit and place of forming the image, the condition found in the second type of instrument described above is obtained, namely, the scattered light from all the material 
surfaces of the instrument is spread equally over the two photometric fields at their dividing line. ${ }^{1}$

Severe tests of this instrument, such as directly viewing incandescent lamp filaments and an acetylene flame, have proved it to have the characteristics for which it was designed, not only when all surfaces are perfectly clean and new, but when purposely covered with dust, to imitate the conditions which might occur after long use.

In working with this spectrophotometer the substitution method was always used, to avoid any errors due to selective absorption in the Brodhun sector, or silvered prism surfaces. This should, of course, always be done in spectrophotometry where accuracy is desired. A possible objection that may be raised to the instrument - that it is impossible to vary the relative intensities by changing two slit openings - may be dismissed on similar ground. The range of slit openings necessary to make matches with sources very different in color is altogether too great for accuracy unless the dispersion is large.

The objections may be made to the discussion above that measurements should never be made with instruments not in perfect condition, and that the errors indicated are small except where the light sources measured are so different that one is less concerned with absolute accuracy than with the relative order of magnitude of the two. The objections are perfectly valid. Nevertheless, under certain conditions, such as the use of portable instruments for daylight measurements, laboratory conditions of cleanliness and ability to shield an instrument from light cannot be obtained. When so used, an instrument which loses its symmetry with the presence of scattered light may give results in error by an indefinite and of ten large amount. Prudence would indicate therefore that when very differently colored sources are compared under. other than the most favorable laboratory conditions an instrument should be used which preserves its symmetry. Or if a form is used in which scattered light destroys the symmetry, the instrument should

\footnotetext{
' In practice it proved well to have the photometric dividing surface quite near the front slit, using a short focused lens. Otherwise the Brodhun sector with its rather small angular opening, cuts the field of vision.
} 
have its diaphragms looked to with especial care, and, for safety, colored glasses should be used as indicated above.

The object of this note has been to call attention to the fact that certain spectrophotometers which are symmetrical when the two sources observed are the same in spectral character, may become unsymmetrical when sources different in character are compared, if scattered light is present. The two types of instrument in which this defect is and is not present, are indicated. An instrument possessing the large monochromatic field of the Lummer-Brodhun and Brace instruments, but which does not become unsymmetrical in the presence of stray light, is described. ${ }^{1}$

Physical laboratory of the National Electric lamp Association, Cleveland, $O$.

${ }^{1}$ Since the construction of the above instrument a description of a similar instrument is given by M. J. Thovert in the Journal de Physique for November, 1909. It is described as a convenient means of altering an ordinary spectroscope to a spectrophotometer and dispensing with the double collimator of the Lummer-Brodhun instrument while obtaining a similar field. No discussion is given, however, of its behavior toward scattered light or the arrangement of image, slit length, etc., to avoid scattered light effects. 